INRA Prod. Anim., 2007, 20 (1), 11-16

\title{
Justifications éthiques des préoccupations concernant le bien-être animal
}

R. LARRÈRE

INRA, UR1216 Transformations Sociales et politiques liés au Vivant, 63/65 Boulevard de Brandebourg, F-94205 Ivry-sur-Seine, France

Courriel :larrere@ivry.inra.fr

«L'intensification de l'élevage a considérablement modifié les conditions de vie des animaux d'élevage. La claustration, les grands effectifs, la restriction de l'espace disponible, l'appauvrissement en stimulations de l'environnement et la perturbation des relations sociales sont devenus la règle [...]. Cette situation est jugée d'autant moins tolérable pour une frange croissante de l'opinion publique, qu'elle est perçue comme étant à l'origine d'une profonde souffrance physique et mentale chez les animaux qui y sont soumis» (Dantzer 2001). Avancé dans un article consacré au bien-être animal, cet argument illustre bien le fait que si les animaux peuvent faire l'objet d'un souci moral, si le traitement qu'on leur fait subir n'est pas un acte neutre, c'est qu'il s'agit d'êtres sensibles. La sensibilité, cette capacité à ressentir (et exprimer) des états mentaux comme la douleur et le plaisir, la souffrance et la satisfaction, commune aux hommes et aux animaux, précède chez les premiers ce qui les distingue des seconds (la parole, la raison, la symbolisation, etc.). Toute une tradition philosophique s'accorde sur ce point, en dépit de Descartes : les animaux n'étant pas des machines, on ne saurait les traiter en purs objets-matières premières ou bien outils de production. Puisque les animaux sont des êtres sensibles, qu'ils ont des émotions, la manière dont ils sont traités leur importe, et devrait donc nous importer. C'est pourquoi il serait justifié, sinon d'accorder à tout animal le droit de ne pas souffrir, du moins de se préoccuper de son bien-être.

\section{1 / Aux sources du bien-être animal}

L'insistance sur la sensibilité, comme la notion même de bien-être, ont pour origine l'utilitarisme, théorie morale qui s'est construite à la charnière des
XVIII gement adoptée, surtout dans les pays de langue anglaise.

L'utilitarisme articule trois propositions fondamentales (Audard 1996) :

1) Il propose un critère du bien et du mal : la souffrance est un mal et le bonheur un bien. Jeremy Bentham, qui en fut le fondateur, assimilait le bonheur au bien-être, ce dernier étant l'état mental qu'expérimente tout individu lorsque ses désirs sont satisfaits.

2) Il comporte un impératif moral : celui de maximiser le bien-être. Chaque individu est présumé rechercher le maximum de bonheur pour le minimum de souffrance : «la nature a placé l'humanité sous l'empire de deux mâ̂tres, la peine et le plaisir. C'est à eux seuls qu'appartient de nous indiquer ce que nous devons faire, comme de déterminer ce que nous ferons. D'un côté le critère du bien et du mal, de l'autre la chaîne des effets et des causes sont attachés à leur trône» (Bentham 1789). Un spectateur impartial reconnaitra aisément à tous les individus le même droit de maximiser ainsi leur bien-être. Il jugera, avec la même impartialité que : «le principe du plus grand bonheur pour le plus grand nombre est la mesure du juste et de l'injuste» (Bentham 1776). Ce faisant, l'utilitarisme conçoit la société comme une simple addition d'atomes, supports de plaisir ou de peine. Mais pour que l'impartialité soit respectée, chaque individu compte pour un - et ne compte que pour un - dans le calcul qui équilibre souffrances et satisfactions dans l'évaluation du «plus grand bonheur pour le plus grand nombre».

3) L'utilitarisme suit enfin une règle de méthode : il pose qu'il faut évaluer les actes à la somme de bien-être qui en découle. Par cette règle, l'utilitarisme s'oppose à toute approche déontologique de l'éthique : ce n'est pas le respect de principes, ou de droits, qui qualifie le caractère moral d'une action, si bien qu'un acte qui pourrait paraître injuste ou condamnable, peut-être justifié si les conséquences en sont bénéfiques.

\section{2 / L'élargissement de l'uti- litarisme aux animaux}

Dès que l'on considère que le plaisir est un bien et la souffrance un mal, le champ de la considération morale doit être étendu à tous les êtres sensibles. Ce fut d'ailleurs le point de vue de Jeremy Bentham (1789) : dans une note, maintes fois citée par les défenseurs de la cause animale, il affirmait ainsi que «La question n'est pas peuvent-ils raisonner? Ni peuvent-ils parler? Mais bien peuvent-ils souffrir?».

En toute logique, l'univers moral de l'utilitarisme ne se réduit donc pas à l'humanité. Mais pendant longtemps les théoriciens de l'utilitarisme n'en tireront aucune conséquence. Il a fallu attendre la fin du XXème siècle pour qu'un philosophe australien, Peter Singer, élargisse explicitement la théorie morale de l'utilitarisme aux animaux : «si un être souffre, il n'y a aucune justification morale qui permette de refuser de prendre en considération cette souffrance. Quelle que soit la nature d'un être, le principe d'égalité exige que sa souffrance soit prise en compte de façon égale avec toute souffrance semblable - dans la mesure où des comparaisons approximatives sont possibles - de n'importe quel autre être. Si un être n'a pas la capacité de souffrir, ni de ressentir du plaisir ou du bonheur, alors il n'existe rien à prendre en compte» (Singer 1993). C'est dire que tous les êtres sensibles - et eux seuls - doivent pouvoir entrer dans les calculs qui évaluent toute action selon le rapport entre son coût (en terme de souffran- 
ces) et ses avantages (en terme de désirs satisfaits).

Pour l'utilitariste, il en est de l'élevage, comme du dressage ou de l'expérimentation : tout traitement des animaux est en principe légitime. Mais pour qu'il soit moralement défendable, il faut que l'augmentation totale de bien-être qui en résulte excède la quantité de souffrances qu'il inflige. Entre deux façons d'utiliser des animaux, sera retenue celle qui maximise le bienêtre. Les animaux (ou les hommes) concernés rentrent simplement dans le calcul du «plus grand bonheur pour le plus grand nombre», et chaque individu (homme ou animal) compte pour un dans cette sommation.

Étendre le monde moral à l'ensemble des êtres sensibles, dans le cadre de cette éthique rencontre en premier lieu le problème de la limite d'une telle extension. Où se clôt l'univers moral élargi de la sorte ? Quelle souffrance animale faut-il prendre en considération ? Se souciera-t-on uniquement du bien-être et de la douleur des mammifères et des oiseaux ? Doit-on aussi se préoccuper des poissons et des escargots ? Pour placer la limite entre les animaux dont la souffrance doit être prise en compte, il faudrait être capable d'appréhender l'univers émotionnel et mental des différentes espèces, ce qui est encore loin d'être le cas : si des recherches sont en cours, l'étude des émotions chez les animaux d'élevage n'est guère plus développée que celle de leurs capacités cognitives (Dantzer 2001, Duncan et Peterich 1991, Boissy et al 2007).

Pour Peter Singer, la capacité de souffrir est une condition nécessaire et suffisante pour avoir des intérêts. Or, le principe utilitariste veut que nous accordions dans nos délibérations morales un poids égal aux intérêts de tous ceux qui sont affectés par nos actions : «le principe de la considération égale des intérêts fonctionne comme une balance, pesant les intérêts de façon impartiale» (Singer 1983). Seuls des préjugés ont ainsi pu conduire les sociétés à accorder plus de poids aux intérêts d'un être qu'à ceux d'un autre. Jadis, par préjugé raciste, les esclavagistes ont systématiquement négligé la souffrance et le bonheur des Noirs. C'est en raison d'un autre préjugé, «l'espécisme», que l'on accorde encore dans la communauté des êtres sensibles, plus de poids aux intérêts des hommes qu'à ceux des animaux et que l'on est choqué de voir un animal compter pour autant qu'un homme. Jeremy Bentham (1789) l'avait, en son temps, remarqué : «Les Français ont déjà réalisé que la peau foncée n'est pas une raison pour abandonner sans recours un être humain aux caprices d'un persécuteur. Peut-être finira-t-on un jour par s'apercevoir que le nombre de jambes, la pilosité de la peau ou l'extrémité de l'os sacrum sont des raisons tout aussi insuffisantes d'abandonner une créature sensible au même sort».

Pour Singer (1993), l'heure serait donc venue, après la libération des esclaves au nom de l'antiracisme, celle des femmes au nom de l'antisexisme, de libérer les animaux au nom de l'antispécisme. Libérer les animaux, c'est d'abord nous libérer d'un préjugé. C'est ensuite intégrer, de manière impartiale, les intérêts des animaux dans l'évaluation des conséquences de nos actions. Concrètement, cela conduit Peter Singer à condamner tout traitement des animaux que l'on estimerait inadmissible, s'il était appliqué à des hommes.

\section{3 / Les recherches concer- nant le bien-être animal}

Les préoccupations qui concernent le bien-être des animaux dérivent incontestablement de l'utilitarisme, mais dans une interprétation moins radicale (et sans doute moins fidèle à Bentham) que celle de Singer. Il ne s'agit pas de mettre tous les êtres sensibles sur un pied d'égalité mais, tout en accordant la primauté aux intérêts humains, de se préoccuper de la façon dont les contraintes que l'on impose aux animaux sont susceptibles de les affecter. Il ne s'agit plus de comptabiliser équitablement le bien-être des hommes et des bêtes, mais de protéger les animaux (parce qu'ils sont faibles et placés sous notre dépendance), des souffrances excessives et inutiles.

C'est dans le contexte d'une critique éthique des formes industrielles de l'élevage contemporain, qu'une communauté scientifique s'est ainsi structurée sous la rubrique de l'Animal Welfare (Larrère 2003, Bourdon 2003). Bien mieux établie dans les pays d'Europe du Nord qu'elle ne l'est en France, cette communauté scientifique associe des éthologistes, des spécialistes de l'univers émotionnel et des aptitudes cognitives des animaux, des neurophysiologistes, mais aussi des philosophes, des théologiens et des spécialistes d'éthique animale. C'est elle qui a traduit les contestations des défenseurs de la cause animale en terme de «bien-être». Elle est parvenue à faire valoir son expertise, tant pour évaluer la réaction des animaux aux contraintes de l'élevage, que pour contribuer à définir des normes de production.

S'il est généralement conçu comme un état mental individuel, trois définitions du bien-être coexistent au sein des recherches qui le concernent (Burgat 2001, Dantzer 2001).

1) Les recherches associées aux organisations interprofessionnelles de la viande ou du lait adoptent volontiers une définition, selon laquelle le bienêtre se réduirait à l'absence de blessure, de maladie, de douleur manifeste. La question pertinente de ce point de vue, n'est pas d'évaluer le bien-être, mais de prendre la mesure de son inverse : il s'agit de savoir si une contrainte donnée imposée aux animaux se traduit (ou non) par une augmentation de la mortalité, de la morbidité, ou des traumatismes. En l'absence de tels signes, on considèrera que le bien-être des animaux est respecté. De fait, ces critères pourraient faciliter l'identification des problèmes auxquels les animaux sont confrontés ; de même pourraient-ils aider à comparer les méthodes d'élevage. L'ennui, comme l'a remarqué Robert Dantzer (2001), c'est que les données épidémiologiques restent trop réduites voire absentes alors qu'elles seraient indispensables pour rendre opérationnelle cette définition minimaliste du bien-être.

2) Dans les instituts de recherche, une seconde conception s'est imposée : elle définit certes le bien-être comme l'absence de souffrance, mais elle considère que l'animal souffre dès lors qu'il ne parvient pas à mobiliser ses ressources physiologiques et/ou mentales pour faire face aux contraintes qui lui sont imposées. Selon Robert Dantzer (2001), la majorité des spécialistes se sont engagés de la sorte dans la recherche d'indicateurs biologiques - ou comportementaux - du stress associé aux difficultés qu'éprouvent les animaux à s'adapter à leur environnement. Il remarque alors que, par construction, ces travaux visent à protéger l'animal de la souffrance, mais qu'ils ne remettent pas en cause les contraintes qui lui sont imposées, ni le modèle de production contraignant auquel il est soumis.

3) Certains éthologistes adoptent une définition plus positive du bien-être : c'est, selon eux, l'état dans lequel se trouve un animal quand il peut «expri- 
mer les comportements naturels de son espèce» ${ }^{1}$; ou bien quand il réalise ses aspirations. L'idée est d'observer, dans un environnement de plein air, le répertoire des comportements qu'expriment les animaux, et de s'en inspirer pour concevoir les modalités de l'élevage. Il ne s'agit donc plus de s'interroger sur l'adaptation des animaux aux contraintes de l'environnement qu'on leur impose, mais d'adapter cet environnement à leur comportement «naturel». Cela conduit à s'interroger sur des dispositifs d'élevage qui assureraient à tout animal «la liberté de mouvement, la possibilité d'explorer le milieu, d'exprimer les comportements propres à l'espèce, d'avoir des relations avec leurs congénères »(Burgat 2001), sans exclure les soins de l'éleveur et tout en tenant compte de ses conditions de travail. Cela signifie-t-il qu'il faille respecter tous les comportements naturels d'une espèce ? Certains comportements «naturels» ne sont pas nécessairement désirables. La fuite devant un prédateur fait partie des stratégies de survie propre à l'espèce, mais elle s'accompagne de stress, non de confort. On ne saurait ajouter des prédateurs dans un élevage pour que le comportement anti-prédateur s'exprime. La question des interactions agonistiques entre animaux est, de ce point de vue, particulièrement délicate : d'une part ces agressions font partie du registre des comportements naturels de l'espèce et d'autre part, elles infligent des douleurs aux animaux qui s'affrontent. Il semble plus raisonnable de favoriser l'expression des comportements naturels pour lesquels il existe une motivation positive de la part de l'animal.

Remarquons que cette troisième définition du bien-être nous fait sortir de la filiation utilitariste. Cette démarche pose un principe auquel doit se conformer toute action technique sur l'animal, le respect des comportements «naturels» de son espèce : tout dispositif d'élevage qui les entrave est donc à écarter. Les éthologistes qui ont adopté cette démarche rejoignent ainsi une position déontologique et s'écartent de l'utilitarisme et de l'évaluation des conséquences de l'action technique en terme de bien-être.

\section{4 / Critique de l'éthique du bien-être}

On peut opposer à l'éthique du bienêtre l'argument de l'intégrité. Peter Sandøe fait ainsi valoir quelques cas intuitivement choquants, et auxquels, cependant, l'éthique du bien-être n'a rien à objecter. Il en est ainsi des bovins Blanc Bleu Belge sélectionnés pour avoir un arrière-train si large qu'ils ne peuvent naître que par césarienne. Or, du point de vue de l'éthique du bienêtre (au moins aux deux premiers sens mentionnés ci-dessus ${ }^{2}$ ), il n'y a rien à objecter : on m'a affirmé un jour (à l'Université libre de Bruxelles) que la mise bas par césarienne apporte du bien-être à la vache ${ }^{3}$. Pourtant, le sens commun s'indigne de telles pratiques. C'est pour donner sens à cette intuition morale, que Peter Sandøe invoque «l'intégrité» ${ }^{4}$.

Selon un manuel de bioéthique du "Centre for Ethics and Law» de Copenhague (Rendtorff et Kemp 2000), «l'intégrité peut se dire de toute chose - entité ou système - qui a sa propre cohérence». Mais l'intégrité n'est un principe moral que lorsqu'il s'agit de l'intégrité d'une entité qui vaut par elle-même, à qui l'on peut attribuer une «valeur intrinsèque». C'est le cas de tout individu auquel on peut reconnaître une «histoire de vie» ou d'une espèce «animale ou végétale, qui a existé dans la nature depuis longtemps et qui tire ainsi sa cohérence de son histoire évolutive» (Rendtorff et Kemp 2000). Pour que la vie d'un individu soit saisie comme une unité cohérente, il faut qu'il ne vive pas uniquement dans l'instant, qu'il soit le sujet d'une vie - subject of a life -, pour reprendre l'expression de Tom Regan (1983) qu'il ait une mémoire du passé, soit capable d'apprentissage, et qu'un horizon d'attente oriente ses actions. Cette identité qui se maintient au cours d'une vie enrichie d'expériences, suppose de toute évidence des capacités cognitives élaborées. Ce sont celles des hommes, et c'est la raison pour laquelle l'intégrité est un principe important en éthique médicale. Tous les êtres vivants n'ont certes pas une telle «histoire de vie», mais il n'est pas exclu que certains animaux disposent approximativement des capacités nécessaires à être ainsi les sujets d'une vie. On songe, bien sûr, à certains mammifères supérieurs (primates, éléphants, cétacés) et aux animaux de compagnie fortement anthropisés. Mais qu'en est-il des vaches, des porcs ou des volailles ?

Pour tous les autres animaux, la cohérence est celle d'une histoire évolutive, non d'une histoire individuelle. Le concept d'intégrité s'applique donc aux lignées, aux variétés, aux espèces. Il peut néanmoins servir de critère pour évaluer telle ou telle intervention. Cela permet, par exemple, de trouver des arguments pour s'opposer à ce que l'on sélectionne du bétail comme le Blanc Bleu Belge (même en supposant que ces animaux n'ont pas «d'histoire de vie» individuelle). Des bovins qui ne peuvent naître sans césarienne ne sauraient survivre en dehors des conditions d'élevage que l'on a programmées pour eux. Ils n'ont d'autre histoire que celle dans laquelle on les a inscrits de force : «l'histoire de vie» de ces animaux est donc hétéronome, faite d'un ensemble de décisions économiques et techniques, et elle produit des animaux qui ne sauraient avoir la moindre postérité sans soins particuliers.

Mais la définition éthologiste du bien-être ne tombe pas sous la critique précédente. La mise bas sans césarienne est un comportement «naturel» chez les vaches. Il n'est donc pas souhaitable, dans cette démarche éthologique, de sélectionner des vaches incapables de mettre bas sans césarienne.

\section{Conclusion : de l'intérêt de la définition éthologique du bien-être}

A la différence des approches inspirées par la tradition utilitariste, la définition éthologique du bien-être animal, outre qu'elle peut répondre aux critiques relatives à l'intégrité, présente l'avantage de ne pas ignorer les réseaux de sociabilité dans lesquels s'insèrent

\footnotetext{
${ }^{1}$ Remarquons qu'il s'agit là de la cinquième des «libertés» qui, selon la Fédération anglaise des Associations de protection animale, doivent servir de règles à l'évaluation du traitement des animaux : absence de maladie, de faim et de soif, de stress, de peur... et possibilité d'exprimer les comportements naturels de l'espèce.

2 Mais nous avons vu que la troisième définition sort de l'éthique du bien-être stricto sensu.

3 Mon contradicteur avançait pour preuve que l'on y a de plus en plus recours en obstétrique humaine pour le confort des mères !

${ }^{4}$ Conférence au premier congrès de l'European Society for Agricultural and Food Ethics, Wageningen, 1999.
} 
les vies animales. Dans l'élevage industrialisé, où il est traité comme une unité numérique ou regroupé en lots homogènes, l'animal est coupé de son environnement social, des relations avec ses congénères, mais aussi avec l'éleveur. Cela conduit à entraver les comportements sociaux «naturels» de l'espèce (relations des mères et de leurs petits, relations hiérarchiques du troupeau). Cela conduit aussi à appauvrir les relations entre les hommes et leurs animaux domestiques.

Les espèces domestiquées sont presque toutes des espèces sociables, avec ce que cela implique de hiérarchisation et de dominance à l'intérieur des groupes sociaux. La domestication ne fut d'ailleurs possible que parce que les hommes surent utiliser à leur profit les rapports de sociabilité existant chez certaines espèces animales : «toutes les créatures qui ont été domestiquées avec succès étaient originellement sociables, elles ont transféré aux êtres humains la confiance et la docilité dont elles faisaient preuve, à l'état sauvage, envers leurs parents, puis adultes envers les animaux dominants de leur meute ou de leur troupeau» (Midgley 1983).

Quels qu'aient été les facteurs ayant favorisé la domestication, les animaux domestiques sont, en quelque sorte, des membres subsidiaires de la communauté humaine. Cela signifie que, s'ils sont soumis à une relation hiérarchique avec l'homme, il y a néanmoins entre eux et ceux qui les élèvent, des échanges de biens, de services et d'affects. Ces relations réciproques se font à l'avantage des deux parties, comme s'il y avait eu une sorte d'arrangement entre les hommes et les animaux dont ils ont cherché le concours.

De tels échanges de services, d'informations et d'affects, sont porteurs d'obligations : c'est ce que nous avons désigné par l'expression de «contrat domestique» (Larrère et Larrère 2000, 2001). Nous avons voulu dire par là que les rapports à l'intérieur de ces «communautés mixtes» (selon 1'expression de Mary Midgley), que les communautés humaines ont formé avec leurs animaux, ne sont pas inscrits dans un ordre naturel préexistant, mais qu'ils résultent d'une histoire. Comme si, par une sorte d'apprentissage mutuel, un accord s'était établi entre les animaux et les éleveurs. Comme si «l'histoire de vie» des animaux domestiqués était tissée de sortes de consentements volontaires, réitérés d'une génération à la suivante, à la forme de «société mixte» ainsi créée. Ces rapports sont réciproques, mais ils sont inégalitaires (gravement inégalitaires, puisqu'ils incluent la possibilité de mise à mort des animaux, et c'est pourquoi ils sont contestés).

Si l'on adopte la troisième définition du «bien-être» animal, on est conduit à prendre en considération les interactions sociales entre animaux (y compris celles qui impliquent des affrontements susceptibles de blesser des individus). De même est-on conduit à se préoccuper des rapports individualisés, subjectifs et personnalisés que les éleveurs peuvent avoir avec leurs animaux.

\section{Références}

Audard C., 2004. Utilitarisme. In : Dictionnaire d'éthique et de philosophie morale. Canto-Sperber M. (Ed), 3 ème édition, P.U.F., Paris, France, 2001-2009.

Bentham J., 1776. A fragment on government. Cambridge University Press, Cambridge, UK, Edition 1988, 128p

Bentham J., 1789. An introduction to the principles of morals and legislation. In : The collected works of Jerely Bentham. F. Rosen, J.H Burns (eds), Clarendon Press, Oxford, UK, Edition de 1983, Chapter XVII, Section I, note 2, 283.

Boissy A., Arnould C., Chaillou E., Colson V., Desiré L., Duvaux-Porter C., Greiveldinger L., Leterrier C., Richard S., Roussel S., Saint-Dizier H., Meneier-Salaün M.C., Valence D., 2007. Emotions et cognition : stratégie pour répondre à la question de la sensibilité des animaux. INRA Prod. Anim., 20(1), 17-22.

Bourdon J.P., 2003. Recherche agronomique et bien-être des animaux d'élevage. Histoire d'une demande sociale. Histoire et Sociétés rurales, 19, 221-239.
Burgat F., 2001. Bien-être animal : la réponse des scientifiques. In : Les animaux d'élevage ont-ils droit au bien-être ? F. Burgat, R. Dantzer (Eds), INRA Editions, Paris, France, 105-133.

Dantzer R., 2001. Comment les recherches sur la biologie du bien-être animal se sont-elles construites ? In : Les animaux d'élevage ont-ils droit au bien-être ? F. Burgat, R. Dantzer (Eds), INRA Editions, Paris, France, 85-103.

Duncan J.H., Peterich J.C. 1991. The implications of cognitive processes for animal welfare. J. Anim. Sci., 69, 5017-5022.

Larrère R., 2003. L'élevage contemporain en question : demande sociale, préférences des consommateurs ou interrogations citoyennes ? INRA Prod. Anim., 16, 329-332.

Larrère C., Larrère R., 2000. Animal rearing as a contract. J. Agric. Env. Ethics, 12, 51-58.

Larrère C., Larrère, R., 2001. L'animal, machine à produire : la rupture du contrat domestique. In : Les animaux d'élevage ont-ils droit au bien-être ? F. Burgat, R. Dantzer (Eds), INRA Editions, Paris, France, 9-24.

Midgley M., 1983, Animals and why they matter, University of Georgia Press, Athens, USA, 158p.

Regan T., 1983. The case of animal rights, Routledge and Kegan Paul, Londres, UK, 425p.

Rendtorff D. J., Kemp P, 2000. Basic ethical principles in european bioethics and biolaw, vol. I Autonomy, dignity, integrity and vulnerability. Report to the European commission of the Biomed-II project, basic ethical principles in bioethics and biolaw 1995-1998, Centre for ethics and law, Copenhagen, Denmark, and Institut Borja de bioética, Barcelona, Spain, $40-45$.

Singer P., 1983. Practical ethics, Cambridge University Press, Cambridge, $2^{\text {nd }}$ Ed., 237p.

Singer P., 1993. La libération animale, (Trad. Fr.), Grasset, Paris, France, 382p.

\section{Résumé}

Les animaux domestiques peuvent-ils faire l'objet d'un souci moral ? Pourquoi devrions-nous nous préoccuper de leur bien-être ? Depuis Jeremy Bentham, l'utilitarisme a étendu la considération morale à tous les êtres sensibles. Cette tradition comporte aussi bien des versions radicales (comme celle de Peter Singer, avec sa thèse de la libération animale) que des acceptions plus modérées (comme les différentes façons de prendre en compte le bien-être des animaux).

Dans les recherches qui le concernent, le bien-être animal est défini de trois façons différentes : $i$ ) l'absence de mortalité et de morbidité ; ii) l'absence de stress ; iii) la possibilité d'exprimer les comportements naturels de l'espèce. Si les deux premières définitions conduisent à négliger l'intégrité des animaux, la troisième présente l'avantage d'en tenir compte et de prendre en considération les besoins et les comportements sociaux des animaux d'élevage. 


\begin{abstract}
Animal well-being and why it matters

Can animals, and especially domestic animals, be the object of moral concern? Should we care about their well-being? In the wake of Jeremy Bentham, Utilitarianism has extended moral consideration to every sentient being, from the most radical position, such as Singer's thesis of animal liberation, to more moderate stances concerned with animal well-being. In scientific research, three definitions of well-being coexist: $i$ ) absence of mortality and morbidity; ii) absence of stress; iii) possibility of expressing natural behaviour. While the first two tend to ignore the integrity of the animals, the third takes it into account and acknowledges the needs and social behaviour of farm animals.
\end{abstract}

LARRÈRE R., 2007. Justifications éthiques des préoccupations concernant le bien-être animal. INRA Prod. Anim., 20, $11-16$. 
